\title{
Carbometallation chemistry
}

\author{
Ilan Marek
}

\section{Editorial}

\section{Address:}

Schulich Faculty of Chemistry, Technion-Israel Institute of

Technology, Haifa 32000, Israel

Email:

Ilan Marek - chilanm@tx.technion.ac.il

Keywords:

carbometallation
Open Access

Beilstein J. Org. Chem. 2013, 9, 234-235.

doi:10.3762/bjoc. 9.27

Received: 24 January 2013

Accepted: 29 January 2013

Published: 04 February 2013

This article is part of the Thematic Series "Carbometallation chemistry".

Guest Editor: I. Marek

(C) 2013 Marek; licensee Beilstein-Institut. License and terms: see end of document.
Following the pioneering Ziegler addition of nucleophiles to nonactivated unsaturated carbon-carbon bonds, the controlled carbometallation reaction has emerged. Since then, reactions that result in the addition of a carbon-metal bond of an organometallic across a carbon-carbon unsaturated system, leading to a new organometallic in which the newly formed carbon-metal bond can be used for further synthetic transformations, are called carbometallation reactions. In the past few decades, the intra- as well as intermolecular additions of various organometallic species to a large variety of alkynes, alkenes and allenes have been successfully reported. Although the carbometallation reaction on alkynes is generally a wellcontrolled and predictable transformation, leading to large variety of substituted stereocontrolled alkenyl metals, the addition of organometallic species to acyclic nonactivated alkenes still represents a formidable and yet unresolved synthetic challenge. Particularly stimulating would be the regio-, stereo- and enantioselective addition of organometallic species on $\alpha, \beta$ disubstituted double bonds, leading to a configurationally stable $\mathrm{sp}^{3}$ organometallic that would subsequently react with electrophiles. This landmark transformation would formally result in the 1,2-bisalkylation of nonactivated alkenes! In this Thematic Series, you will find excellent contributions tackling various problems of carbometallation reactions, indicating a lively and rapidly moving field, and I have no doubt that more elegant transformations of $\mathrm{C}-\mathrm{C}$ unsaturated bonds will continue to appear, including the enantioselective carbometallation reaction of substituted nonactivated alkenes. I would like to warmly thank all the contributors of this Thematic Series that have beautifully highlighted the state of the art of the field.

I have tremendously enjoyed reading this Thematic Series and I am convinced that you will all share in this pleasure!

Ilan Marek

Haifa, January 2013 


\section{License and Terms}

This is an Open Access article under the terms of the Creative Commons Attribution License

(http://creativecommons.org/licenses/by/2.0), which permits unrestricted use, distribution, and reproduction in any medium, provided the original work is properly cited.

The license is subject to the Beilstein Journal of Organic Chemistry terms and conditions:

(http://www.beilstein-journals.org/bjoc)

The definitive version of this article is the electronic one which can be found at:

doi:10.3762/bjoc. 9.27 